\title{
LOS SIGLOS XVI Y XVII
}

\author{
Óscar Mazín \\ El Colegio de México
}

\begin{abstract}
A lo largo de sus 70 años Historia Mexicana es atalaya indiscuA tible de la historiografía. La continuidad de sus cuatro números anuales la convierte en fuente imprescindible para tomar el pulso a los procesos de profesionalización de nuestra disciplina. En ocasión de este feliz homenaje, se me ha pedido dar cuenta de lo publicado sobre los siglos xvi y xvII. Es un encargo complejo de extensión limitada, lo que hace necesario apretar la síntesis.

Hay que preguntarse cuántos artículos salieron publicados, en qué décadas de la revista se advierte más producción y en cuáles menos. También interesa saber si la mayor concentración de materiales permite distinguir etapas. ¿Cuál es la tónica o carácter de cada una? ¿Qué temas predominan? ¿Qué se puede decir de la procedencia de los autores? ¿Qué regiones son más estudiadas? ¿Hay trabajos relativos a otras posesiones de la corona de España? Es asimismo importante saber qué tanto ha interesado la historia comparada. ¿Hay artículos que abrazan de manera explícita alguna perspectiva de método? ¿Cómo interactúan los autores con las humanidades y con las ciencias sociales?1
\end{abstract}

\footnotetext{
${ }^{1}$ Para todo ello, el equipo editorial de Historia Mexicana me ha dotado de una base de datos provista de enlaces electrónicos con cada uno de los 138 artículos.
} 
No se puede responder a casi nada de esto sin una apreciación estadística básica. A lo largo de su historia, el total de artículos que en Historia Mexicana tratan acerca de los siglos xvi y xvir es de 138. Se lleva las palmas el siglo Xvi con 67 (48.55\%). Le sigue el xVII con 47 (34.05\%). Sin embargo, 24 artículos (17.39\%) se ubican en la transición entre ambas centurias, por lo que integran un tercer indicador que equilibra el predominio de los textos dedicados exclusivamente al siglo xvi. Hay que aclarar que estos últimos no incluyen los procesos de las conquistas hispanas, que son objeto de otro capítulo en este homenaje.

¿Cómo está distribuida esa producción? Curiosamente, la más numerosa se ubica en el extremo inaugural de la revista. En cambio, la de mayor concentración por años corresponde a los primeros quinquenios del siglo xxi. Se perfilan, pues, claramente, dos etapas: La inicial (1951-1970), que reúne 56 artículos, o sea $40 \%$ del total. Y en el otro extremo (2001-2016), 35 artículos, que equivalen a $25.3 \%$ del conjunto. ${ }^{2}$ Ambas concentran poco más de $65 \%$ de la producción aquí considerada. El resto se halla disgregado a lo largo de las décadas intermedias: a la de 1970 corresponden 12 artículos; durante los años ochenta aparecieron 24, y en la década de 1990 se publicaron 10 trabajos.

\section{UNA AURORA HUMANÍSTICA}

Acerquémonos, pues, a la etapa fundacional. Nunca la historia "colonial" ha interesado más a los autores. Para explicarlo hay que considerar algunos hechos. El mundo de la posguerra, tan atraído por la "descolonización", debió ser un estímulo relevante para indagar qué clase de posesiones habían sido las del

Fue elaborada de manera comprehensiva y acuciosa. Hay que agradecerlo a Frida Martínez León y a mi querida e inolvidable Beatriz Morán Gortari. Las referencias a pie de página de este artículo se refieren a Historia Mexicana.

${ }^{2}$ De esos 35 artículos, 14 tratan de los siglos Xvi y Xviı; 8 sólo de este último, y 13 se refieren al primer siglo de Nueva España. 
Nuevo Mundo y Nueva España en particular. Pero, además, en México había una fascinación por el oficio. Escribir historia se vinculaba a las humanidades, pues había que elaborar explicaciones comprehensivas, de tal manera que no se tendía a la especialización extrema. La gente de letras, historia, filosofía y arte se complacía en hurgar en lo propio para encontrarlo ya fuera en el pasado prehispánico, en el pasado hispánico o "colonial”, o en la epopeya más o menos reciente de un pueblo en armas. También debemos considerar el ímpetu y las aportaciones de aquellos docentes y escritores peninsulares "transterrados" a Hispanoamérica y que en nuestro país fueron acogidos por $\mathrm{La}$ Casa de España/El Colegio de México y por la Universidad Nacional. Sin olvidar que en aquella misma coyuntura (1938-1941) se habían fundado el Centro de Estudios Históricos del primero y los Institutos de Investigaciones Históricas y Estéticas en la segunda. Sus profesores más ilustres descuellan en la época inicial de Historia Mexicana.

Las décadas de 1950 y 1960 se hacen notar por la aparición de trabajos pioneros sobre temas que abrieron brecha y suscitaron debates importantes, algunos todavía vigentes: por ejemplo, el colapso demográfico de la población autóctona en el centro de Mesoamérica, su duración y los inicios de su recuperación. ${ }^{3} \mathrm{O}$ bien, corolario del anterior, la controversia sobre la legitimidad de la conquista hispana y la naturaleza de los indios, con trabajos en el ámbito de las ideas, obras y trayectoria de fray Bartolomé de Las Casas. ${ }^{4}$ La relevancia de estos temas no debe, sin embargo, opacar la riqueza de aquella alborada.

Así tenemos que si la despoblación autóctona es importante, no es menos relevante el estudio de las tendencias de la

3 Borah, "La despoblación del México central” (45), 1962, pp. 1-12 y MiranDA, "La población indígena" (46), 1962, pp. 182-189.

4 O’Gorman, "La idea antropológica" (63), 1967, pp. 309-319; Silva Tena, "Las Casas, biógrafo" (16), 1955, pp. 523-543 y "El sacrificio humano” (63), 1967, pp. 341-357; OrTEGA, "El indio absuelto" (16), 1955, pp. 477-505. 
emigración hispana y el poblamiento a partir de la península ibérica, según regiones de procedencia y sitios de destino. ${ }^{5} \mathrm{El}$ ámbito de la cristianización de los indios dio lugar a trabajos acerca de sus figuras señeras, como fray Juan de Zumárraga; pero también a la influencia que recibieron de las ideas de Erasmo de Rotterdam, sin duda como reflejo del impacto que entonces tuvo Erasmo y España de Marcel Bataillon, obra traducida al español por Antonio Alatorre y publicada en México en $1956 .^{6}$

La conciencia de los españoles oriundos del Nuevo Mundo o "criollos" interesaba entonces más desde una perspectiva filológica y literaria, es decir, a causa de los escritos tanto en prosa como en verso de algunos de sus exponentes. Este enfoque es revelador de los intercambios prevalecientes entre el Centro de Estudios Históricos y el de Estudios Lingüísticos y Literarios de El Colegio de México, todavía bajo la égida de Alfonso Reyes y Raimundo Lida. Interesa la pluma de los miembros de la primera nobleza mexicana, como Juan Suárez de Peralta (Tratado del descubrimiento de las Indias y su conquista), Baltasar Dorantes de Carranza (Sumaria Relación de las cosas de la Nueva España), Juan de Cárdenas (Problemas y secretos maravillosos de las Indias); ${ }^{7}$ la indagación acerca de los autores clásicos que inspiraron la prosa a la vez sobria y grandilocuente de Francisco López de Gómara (Historia general de las Indias y la conquista de México); ${ }^{8}$ o bien el carácter compendioso de la Monarquía

\footnotetext{
5 Boyd-Bowman, “La emigración peninsular” (50), 1963, pp. 165-192 y "La procedencia de los españoles” (65), 1967, pp. 37-71.

6 Bataillon, "Zumárraga reformador” (9), 1953, pp. 1-10; Almoina, "Citas clásicas” (11), 1954, pp. 391-419; MirandA, “Renovación cristiana” (1), 1951, pp. 22-47; Corona, “Religiones indígenas” (40), 1961, pp. 557-570; LóPEz, “Términos del nahuallatolli” (65), 1967, pp. 1-36.

7 Benítez, “Los criollos del siglo Xvi” (2), 1951, pp. 251-267; URANGA, “Juan de Cárdenas” (64), 1967, pp. 477-497; López Cámara, “La conciencia criolla” (23), 1957, pp. 350-377.
}

8 Durand, “Gómara” (6), 1952, pp. 210-222. 
Indiana de fray Juan de Torquemada como crónica. ${ }^{9}$ También se aprecian procedimientos filológicos para desentrañar la autoría de testimonios documentales tan relevantes como la Relación de Michoacán. ${ }^{10} \mathrm{La}$ impronta humanista de esa primera hora alcanza una de sus cimas con el ensayo "El sueño de un sueño". Con penetración y belleza, su autor hace un análisis filosófico y literario de la obra Primero sueño de sor Juana Inés de la Cruz. ${ }^{11}$

Historia Mexicana también acogía artículos de historia del arte. Era el momento de las grandes síntesis sobre el arte colonial y la catedral de México de Manuel Toussaint, así como del arte hispanoamericano por Diego Angulo Íñiguez. El binomio arte e historia gozaba, pues, de vigencia. Los de la revista son trabajos que hacen la crítica a atribuciones de autoría de obras de pintura mural y de retablos en conventos e iglesias del siglo xvi; ${ }^{12}$ o bien discurren acerca de si hubo o no un retrato verosímil de sor Juana. ${ }^{13}$ Además de hacer gala de su oficio, sus autores explican las implicaciones históricas de las obras de arte. Otros se interesan por la historia de actitudes y de costumbres asociadas a los objetos artísticos, como el lujo y el boato como estilo de vida, o bien dan cuenta de actividades artesanales como la herrería y la cerrajería. ${ }^{14}$

Por otra parte, se corrobora una conciencia creciente de la inserción de Nueva España en una primera economía de proporciones mundiales. Es la minería la actividad que la preside, ya sea por medio de la circulación de las monedas de plata acuñadas en México, de la búsqueda del azogue o de las innovaciones

\footnotetext{
9 Moreno, "Vindicación" (48), 1963, pp. 497-515.

10 Bravo, "La Relación" (45), 1962, pp. 13-25.

11 GAOs, "El sueño" (37), 1960, pp. 54-71.

12 Toussaint, “Las pinturas murales" (2), 1951, pp. 173-184 y Castro MoRALES, "El retablo" (79), 1968, pp. 179-189.

${ }^{13}$ De la Maza, "Primer retrato" (5), 1952, pp. 1-23.

14 Durand, "El lujo" (21), 1956, pp. 59-74; Muro Arias, "Herreros y cerrajeros” (19), 1956, pp. 337-372.
} 
tecnológicas en materia de extracción y beneficio de ese metal. ${ }^{15}$ Pero le hace competencia la difusión de colorantes como la grana cochinilla y el "pastel” a partir de plantas tintóreas; ${ }^{16}$ o la introducción de la sericultura en la Mixteca ${ }^{17}$ y de la ganadería extensiva; ${ }^{18}$ sin faltar la ruta transpacífica del galeón de Manila hacia las Filipinas. ${ }^{19}$ Vemos asimismo aparecer una primera comparación sistemática entre las economías de Nueva España y el Brasil. ${ }^{20}$

Un artículo de importante calado historiográfico debe mencionarse por su visión, precoz a la vez que honda, de las fuerzas geopolíticas que estructuraban la Nueva España. Es aquel que explica la centralidad del puerto de Veracruz y su relevancia universal en la transición del siglo xvi al XVII. ${ }^{21}$ También hacen acto de presencia trabajos sobre la movilidad de fronteras hacia el septentrión, ya sea en el ámbito de la guerra chichimeca o de la posterior rebelión tepehuana. ${ }^{22}$ Interesan asimismo los procesos de fundación de ciudades como Durango o la implantación de gobiernos en las provincias ganadas a los indios de guerra para la década de 1590, como San Luis Potosí. ${ }^{23}$

15 Beltrán, "Primeras casas" (3), 1952, pp. 372-394; LANG, "La búsqueda" (72), 1969, pp. 473-484; Muro, “Bartolomé de Medina” (52), 1964, pp. 517531; McMaster, “Aventuras asiáticas” (31), 1959, pp. 372-399.

16 Berthe, "El cultivo del pastel” (35), 1960, pp. 340-377; HeErs, “La búsqueda de colorantes” (41), 1961, pp. 1-27.

17 BoraH, "El origen de la sericultura" (49), 1963, pp. 1-17.

18 Matesanz, “Introducción de la ganadería” (56) 1965, pp. 533-566.

19 Carrera, “La Nao de China” (33), 1959, pp. 97-118; Quirino, “El primer mexicano en Filipinas" (54), 1964, pp. 250-260; Pizano y SAUCEdo, "El puerto de la Navidad” (54), 1964, pp. 227-249; GonzÁlez, "Expansión de Nueva España” (54), 1964, pp. 206-226; Bernal, “México en Filipinas” (54), 1964, pp. 187-205.

20 Mauro, “México y Brasil” (40), 1961, pp. 571-587.

21 Chaunu, "Veracruz" (36), 1960, pp. 521-557.

22 McCarty, “Los franciscanos” (43), 1962, pp. 321-360.

23 Gallegos, "El fundador de Durango" (50), 1963, pp. 272-276; BoraH, "Un gobierno provincial” (52), 1964, pp. 532-550 y "La defensa fronteriza" 
Aun cuando la mayoría de los autores de esta primera etapa interactúan con el resto de las humanidades, no adscriben su trabajo de manera explícita a algún paradigma de método. La interlocución más notoria con las ciencias sociales llegó a Historia Mexicana partir de la década de 1970, ¿Qué es, entonces, lo más característico de los trabajos inaugurales? Definitivamente, la amplitud de miras. Quienes escriben se interesan no sólo por la historia, sino por la literatura, la filosofía, la filología y el arte. Son gente con vocación humanística, escriben bien y saben dirigirse a un público general de lectores ávidos. Si bien estudian Nueva España, su mirada trasciende los límites de ésta en busca de ejemplos, por lo menos en el resto de Hispanoamérica.

Las procedencias de esos maestros fundadores son diversas: hay profesores del exilio español en México, como José Gaos y José Miranda; exponentes de la historiografía francesa, como Pierre Chaunu y Jean-Pierre Berthe; mentores de la Escuela de Berkeley como Woodrow Borah; docentes mexicanos como Edmundo O'Gorman y Silvio Zavala, cuyas obras eran ya referente obligado. También se publicaron trabajos de algunos de sus alumnos, como el de un joven Luis González; en fin, hacen acto de presencia académicos por entonces recién egresados, como Juan Ortega y Medina o Enrique Florescano.

\section{FERMENTO AL FILO DE LOS AÑOS}

La producción de Historia Mexicana acerca de los siglos XvI y xvII comprendida entre 1971 y 2000 equivale a poco menos de $35 \%$ del total aquí examinado. Pero sucede que ahora los artículos no se concentran en un conjunto determinado de años; tampoco configuran propiamente una etapa, pues se hallan disgregados a lo largo de esas décadas. Por este motivo, para

(61), 1966, pp. 15-29. 
identificar temas, tendencias y eslabonarlos, hay que proceder en orden cronológico.

La década de 1970 se significa por entregas de investigaciones en curso o recién consolidadas. Ahora sí se advierte una fuerte interlocución con la demografía, la economía, así como con miradas sociológicas a la "estructura social" en regiones bastante delimitadas, por ejemplo, los minerales ubicados entre San Luis Potosí y Zacatecas. ${ }^{24} \mathrm{O}$ bien, que cuantifican el ganado menor y calculan el peso del comercio hispano en las Mixtecas a expensas de la población y la economía india declinantes. ${ }^{25}$

También encontramos artículos que se interesan por las corporaciones, es decir, por "instituciones" de índole eclesiástica tales como conventos de monjas y cofradías de renombre de los que se describe su fundación y prácticas. No obstante, prevalece la atención a sus haberes, a su modus operandi: cuantificación de haciendas y ganados, de censos al quitar, cuotas y limosnas. ${ }^{26}$ Hay asimismo trabajos sobre una religiosidad asociada a la presencia cada vez más importante de los grupos resultantes de la mezcla de calidades, sobre todo mestizos y mulatos. ${ }^{27} \mathrm{En}$ continuidad con la década anterior, sobresalen artículos sobre milenarismo y escatología franciscana, pensamiento erasmista, gente de saber y ciencia, ya fueran eclesiásticos o seglares vinculados a las matemáticas o a la herbolaria. ${ }^{28}$

Mención especial requieren los textos de la década de 1970 centrados en la historia urbana. Algunos, acaso, se habrán beneficiado del seminario animado por Alejandra Moreno Toscano desde el entonces llamado Departamento de Investigaciones

\footnotetext{
24 Carmagnani, “Demografía y sociedad” (83), 1972, pp. 419-459.

25 Romero Frizzi, “Los intereses españoles” (114), 1979, pp. 241-251.

26 Lavrin, “El convento de Santa Clara” (97), 1975, pp. 76-17 y "La congregación de San Pedro” (116), 1980, pp. 562-601.

27 López Sarrelangue, “Mestizaje y catolicismo” (89), 1973, pp. 1-42.

28 Frost, "El milenarismo" (101), 1976, pp. 3-28; Trabulse, "El erasmismo" (110), 1978, pp. 224-296 y “Un científico mexicano” (93), 1974, pp. 36-69.
} 
Históricas del INAH. Descuellan artículos pioneros sobre las congregaciones de indios de Nueva España con un fuerte componente de geografía histórica, sin olvidar instituciones y formas de tenencia de la tierra. Otros tratan de la fundación de ciudades como Puebla de los Ángeles o del emplazamiento espacial de la capital de Nueva España. Hay trabajos también iniciales acerca del impacto de la venalidad como estrategia fiscal de la corona de España sobre los cabildos o ayuntamientos. ${ }^{29}$

La década de 1980 fue más abundante en artículos, en parte a causa del homenaje a Silvio Zavala en ocasión de sus 80 años de vida, del que resultaron dos números conmemorativos de Historia Mexicana (1989). En algunos títulos se advierte cierta concatenación de intereses respecto a la década anterior. Así, por ejemplo, la evolución de las economías india e hispana, tanto en la Mixteca costera como en la Alta, adopta una posición crítica ante la "crisis del siglo xvir", importante debate internacional de esos años; $; 0$ o la situación que guardaban el comercio y los mercaderes de Guatemala en relación con los de México. ${ }^{31}$ También persiste el tema de la expansión hacia el Pacífico y las Filipinas a partir de Nueva España. ${ }^{32}$ El interés por las formas de trabajo incluye un texto sobre Hernán Cortés como empresario y amo de esclavos negros; ${ }^{33}$ otros se hacen cargo de la mano de obra en los obrajes y del repartimiento forzoso de indios. ${ }^{34}$ En unos y otros repercute la obra portentosa de Zavala.

29 Hirschberg, "La fundación de Puebla" (110), 1978, pp. 185-223; AlvaraDo, "El cabildo y regimiento" (112), 1979, pp. 489-514; GERHARD, "Congregaciones de indios" (103), 1977, pp. 347-395; Boyer, "La ciudad de México" (115), 1980, pp. 447-471.

30 TAKahashi, "De la huerta a la hacienda" (121), 1981, 1-78; Romero Frizzi, "Evolución económica” (128), 1983, pp. 496-526.

31 De la Peña, “Comercio y poder" (120), 1981, pp. 469-505.

32 García-Abasolo, "La expansión mexicana” (125), 1982, pp. 55-88.

33 Verlinden, "Cortés como empresario" (159), 1989, pp. 771-778.

34 Viqueira, "El significado de la legislación” (137), 1985, pp. 33-58; JimÉNEZ Pelayo, "Condiciones del trabajo" (151), 1989, pp. 455-470. 
En la estela de Gibson, Lockhart y Taylor, son numerosos los estudios sobre los indios, sus pueblos, las formas de gobierno y fiscalidad (tributos) tanto en la cuenca de México (Xochimilco) como en los Altos de Chiapas y en Yucatán. ${ }^{35}$ También resalta el estudio y edición de documentos híbridos de mediados del siglo xvI, con pictografías de origen prehispánico y glosas en español para tasar los tributos por orden del virrey Luis de Velasco en pueblos de la cuenca del alto Balsas. ${ }^{36} \mathrm{El}$ homenaje a Silvio Zavala también trajo un texto análogo referente al Alto Perú (Oruro). ${ }^{37}$

Durante los años ochenta reaparece el tema del presunto milenarismo entre los franciscanos; ${ }^{38} \mathrm{el}$ interés por la elaboración de instrumentos de catequesis: doctrinas o catecismos, prontuarios de teología moral y manuales para párrocos..$^{39}$ Rebrotan estudios en torno al pensamiento y tradición de Las Casas; análisis cuidadosos sobre fray Jerónimo de Mendieta en memoriales dirigidos a Juan de Ovando, presidente del Consejo de Indias acerca de lo que ese fraile cronista consideraba que debía ser la "República de los indios"; ${ }^{40}$ o referentes a escritos incendiarios como los de fray Alonso de Maldonado que, al impugnar los justos títulos de la soberanía del Rey Católico en las Indias, incluso suscitaron causas de índole inquisitorial. ${ }^{41}$

En el ámbito del seminario de historia de la educación auspiciado por el Centro de Estudios Históricos de El Colegio de México, destacan aportaciones sobre la influencia social de los

35 Pérez Zevallos, “El gobierno indígena” (132), 1984, pp. 445-462; Gosner, “Las élites indígenas” (132), 1984, pp. 405-423; QuEZADA, "Encomienda, cabildo y gubernatura” (136), 1985, pp. 662-684.

36 Noguez, “Tres documentos pictográficos” (141), 1986, pp. 5-48.

37 SÁnchez-Albornoz, “Territorio y etnia” (153), 1989, pp. 167-179.

38 WeCKMANN, “Las esperanzas milenaristas” (125), 1982, pp. 89-105.

39 De la Torre, “Aspectos sociales de los instrumentos” (152), 1989, pp. 609-622.

40 Assadourian, “Memoriales de fray Gerónimo” (147), 1988, pp. 357-422.

41 Assadourian, “Fray Alonso de Maldonado” (152), 1989, pp. 623-662. 
jesuitas y los métodos de enseñanza en los colegios de la Compañía de Jesús de Nueva España. ${ }^{42}$ También interesa la vida conventual mercedaria como ámbito propicio, aunque no exclusivo, para la formación de sabios como el matemático fray Diego Rodríguez. ${ }^{43} \mathrm{El}$ interés por el saber se extiende hasta el palacio real, ya que un autor escudriña los títulos de la biblioteca del virrey Martín Enríquez. ${ }^{44}$ Era un saber en que el derecho ocupaba un lugar de preeminencia, como testimonia la indagación y discusión acerca de las prerrogativas del reino de Nueva España para hacerse representar en las Cortes de Castilla, así como para convocar a Cortes o "congresos" en sus propios términos territoriales. ${ }^{45}$

La producción de la década de 1990 es poco numerosa y heterogénea en cuanto a temáticas. Hay primicias de trabajos de tesis dirigidos por profesores del Centro de Estudios Históricos de El Colegio y de otras instituciones, aun extranjeras. ${ }^{46}$ Con todo, los trabajos apuntan en dirección tanto a herencias como hacia continuidades respecto de la etapa subsiguiente. Entre las primeras sobresalen textos insertos en el ámbito de la historia regional, especialmente de índole económica: por ejemplo, el impacto del comercio sevillano con la península de Yucatán por el puerto de Campeche; ${ }^{47}$ o los componentes demográficos como sustento para considerar y cuantificar los empalmes de minería y agricultura, así en Guadalajara, las villas y pueblos de su entorno, como en aglomeraciones más lejanas en

\footnotetext{
42 Gonzalbo, "La influencia de la Compañía” (126), 1982, pp. 262-281 y "Paideia cristiana" (131), 1984, pp. 185-213.

43 Trabulse, "La vida conventual” (152), 1989, pp. 743-769.

${ }^{44}$ Намре, "La biblioteca del virrey" (142), 1986, pp. 251-271.

45 Lohmann, "Notas sobre la presencia" (153), 1989, pp. 33-40; Bernal, "Las características” (152), 1989, pp. 663-675.

46 Calvo, "Demografía y economía” (164), 1992, pp. 579-613; Martínez Baracs, "Colonizaciones tlaxcaltecas" (170), 1993, pp. 195-250; QuezadA, "Encomienda, cabildo y gubernatura" (136), 1985, pp. 662-684.

47 García Bernal, "El afianzamiento" (198), 2000, pp. 201-269.
} 
el reino de Nueva Galicia, por ejemplo, Lagos y aun la remota Zacatecas. ${ }^{48}$ Otro tema asociado a etapas anteriores es el relativo a religión y justicia, política y corporaciones eclesiásticas. Reaparece el viejo tópico de fray Bartolomé de Las Casas, esta vez como obispo de Chiapas en sus relaciones no siempre cordiales con sus homólogos, especialmente el de Guatemala. ${ }^{49}$ La justicia en el foro eclesiástico es el ámbito de una monja de Michoacán cuya causa llegó al tribunal ad hoc llamado provisorato. ${ }^{50}$ Un estudio más trata de los ritos idolátricos en pueblos de indios nahuas y zapotecos a partir del análisis de tratados que los denunciaban, como el de Hernando Ruiz de Alarcón. ${ }^{51}$ Los indios de Tlaxcala son objeto de un estudio que los caracteriza como conquistadores y pobladores a lo largo de varias décadas, que sigue sus andares por diversos horizontes: en la Gran Chichimeca y más allá, en el Septentrión de Nueva España, así como en dirección del istmo centroamericano. ${ }^{52}$

Algunos artículos apuntan a la década siguiente, sobre todo en el sentido de que miran más allá de Nueva España. De esta suerte, se indaga la trayectoria de aquellos virreyes de Nueva España que pasaron enseguida a gobernar el Perú, aunque con la particularidad de haber antes ejercido como asistentes en Sevilla, el cargo más importante de designación real inserto en el ayuntamiento de esa urbe del Guadalquivir. ${ }^{53}$ Otro texto examina el tema de la población negra y sus tendencias al mestizaje en Centroamérica, según lo vieron cronistas extranjeros como el inglés Thomas Gage. ${ }^{54}$ Por último, está considerado un grupo

\footnotetext{
48 Calvo, “Demografía y economía” (164), 1992, pp. 579-613.

49 Assadourian, “Fray Bartolomé de Las Casas” (159), 1991, pp. 387-451.

50 Traslosheros, “Los motivos de una monja” (188), 1998, pp. 735-766.

51 TAvÁrez, “La idolatría letrada” (194), 1999, pp. 197-252.

52 Martínez Baracs, “Colonizaciones tlaxcaltecas” (170), 1993, pp. 195-250.

53 Hampe, “Esbozo de una transferencia” (161), 1991, pp. 49-81.

54 PAstor, “De moros en la costa a negros” (174), 1994, pp. 195-235.
} 
de jesuitas de origen flamenco que ejercieron como misioneros en el noroeste de Nueva España. ${ }^{55}$

\section{FORMULACIÓN DE NUEVOS LENGUAJES}

Ya advertí que se perfila una etapa en los primeros lustros del presente siglo (2001-2016). La concentración y densidad de los trabajos permiten asignar al conjunto un título, "Nuevos lenguajes". Sus autores no parecen haber escogido el siglo sobre el cual iban a trabajar de manera prioritaria, sino que discurren en la continuidad de las temporalidades. De ahí que poco más de la mitad de los artículos cuyo tema se ubica en la transición del siglo XVI al XVII correspondan a esta etapa. Los enfoques han ganado en complejidad. Una autora dice adherirse a la "historia cultural" como paradigma de método, otros se pronuncian por una historia "postnacional" ${ }^{56}$ Efectivamente, los intereses toman distancia de la historia nacional como marco exclusivo de referencia. Se hallan estimulados por métodos y especialidades que les hacen atisbar otros horizontes. Más aún, hay trabajos que inscriben a la Nueva España en la perspectiva analítica de las monarquías compuestas de las que formó parte, que fueron su ámbito natural.

Esta situación se hace eco de un proceso fuerte de internacionalización de la disciplina, simultáneo al de globalización del planeta. Su motor ha sido el intercambio entre colegas, intensificado a partir de mediados de la década de 1990 entre universidades y centros de investigación europeos, estadounidenses e iberoamericanos. En el contexto mexicano intervienen autores no solamente de El Colegio y la UNAM, sino de los centros de

55 De Groof, “Encuentros discordantes” (187), 1998, pp. 537-569.

56 Respectivamente, Silva Prada, "Estrategias culturales" (209), 2003, pp. 5-63; Ruiz Ibáñez y Vallejo Cervantes, "Vivir 'sin dexar parte”" (243), 2012, pp. 1109-1170. 
investigación fundados en las décadas precedentes tanto en la capital como en las entidades federativas, como los Colegios, el primero de los cuales en tiempo es el de Michoacán (1979).

Uno de esos nuevos lenguajes corresponde a la resignificación de la que ha sido objeto la historia del derecho. De esta suerte, las "instituciones" o corporaciones se entienden como cuadros o referentes formales del orden social de las que se enfatiza su naturaleza jurídica. Hay trabajos que consideran instituciones seculares o profanas, como los consulados de comercio. ${ }^{57}$ Pero son más numerosos los que indagan en las de índole eclesiástica, lo que obedece a la renovación también efectuada desde los años ochenta y la década siguiente en el ámbito de la historia de la Iglesia católica y su enorme incidencia social en aquellos siglos. Esos estudios presentan un amplio abanico de intereses: pueden referirse a la familia de un obispo y aportar elementos para la biografía como subgénero; ${ }^{58}$ interesarse en prácticas como la impartición de justicia en las repúblicas de indios; ${ }^{59}$ tratar acerca de la dimensión jurídica de la consciencia en el sacramento de la confesión; ${ }^{60}$ preguntarse sobre las devociones en los pueblos y santuarios; ${ }^{61}$ caracterizar el perfil de las órdenes religiosas no mendicantes que llegaron a Nueva España después del Concilio de Trento; ${ }^{62}$ reconsiderar los colegios primitivos, como el de la Santa Cruz de Santiago Tlatelolco; ${ }^{63}$ o, incluso, dar a conocer formas de fiscalidad que antes no interesaban, como las bulas

57 Del Valle Pavón, “Expansión de la economía mercantil” (203), 2002, pp. 517-557.

58 Irigoyen, "Un obispado para la familia” (230), 2008, pp. 557-594.

59 Owensby, “Pacto entre rey lejano” (241), 2011, pp. 59-106.

60 Lira, “Dimensión jurídica de la conciencia” (220), 2006, pp. 1139-1178.

61 RaGon, "Los santos patronos de las ciudades” (206), 2002, pp. 361-389; Moro, “Los santuarios novohispanos” (264), 2017, pp. 1750-1818.

62 Ramírez Méndez, “Las nuevas órdenes religiosas en las tramas” (251), 2014, pp. 1015-1075.

63 Alberro, “El Imperial Colegio de Santa Cruz” (253), 2014, pp. 7-63. 
de la Santa Cruzada o los donativos que la corona de España recaudaba en sus posesiones americanas. ${ }^{64}$

Permitió a los académicos incursionar en todo esto la resignificación de conceptos tales como justicia y jurisdicción. Y como en todo el orden social interactuaban numerosas jurisdicciones, el conflicto nunca tuvo en aquellos siglos un carácter excepcional, sino que fue la norma. De esta suerte surgen nuevos actores, por ejemplo, los defensores de tribunales indios o los procuradores de ciudades y demás cuerpos sociales ante la corte del rey. ${ }^{65}$

Un par de artículos se adscribe al enfoque o especialidad de la historia cultural, entendida como una incursión en el estudio de las formas de representación de los grupos sociales. Entran aquí desde la discursividad relativa al saber, en particular la medicina y la botánica, hasta aquella asociada a las formas de resistencia violenta, como el tumulto o la rebelión en perspectiva comparada entre el Perú y la Nueva España. ${ }^{66}$ Pero también interesan las prácticas judiciales desde el punto de vista de la parafernalia del ceremonial, de los ritos practicados y de los recursos retóricos que intervenían en el recibimiento de grandes dignatarios, lo que explica una nueva vigencia de la emblemática. ${ }^{67}$ Como apunté, los casos no se limitan ya a Nueva España, sino, por ejemplo, miran hacia la corte de Lima, capital de las Indias meridionales. ${ }^{68}$

Un campo más de "nuevos lenguajes" es aquel mediante el cual Historia Mexicana ha respondido a los estudios sobre

\footnotetext{
${ }^{64}$ Respectivamente, Martínez López-Cano, "La administración de la bula" (247), 2013, pp. 975-1017; Grossmann, "La Iglesia al servicio de la fiscalidad" (266), 2017, pp. 551-604 y DE NARDI, "El carácter voluntario de los donativos" (268), 2018, pp. 1535-1596.

65 Cunill, "Los defensores de indios" (246), 2012, pp. 551-590; TraslosheROs, "El tribunal eclesiástico y los indios" (203), 2002, pp. 485-516.

${ }^{66}$ Silva Prada, "El uso de los baños" (205), 2002, pp. 5-56; Salinero, "Rebeliones coloniales” (255), 2015, pp. 895-936.

67 CÁrdenas Gutiérrez, "El teatro de la justicia” (220), 2006, pp. 1179-1220.

68 Osorio, "La entrada del virrey" (219), 2006, pp. 767-831.
} 
las monarquías de España y Portugal, renovados en el ámbito internacional también a partir del final de la década de 1990.69 Entendidas como entidades mundiales de naturaleza compuesta previas a los estados nacionales, en ellas se establecieron y desarrollaron reinos como Nueva España. Había, pues, que salir de los marcos nacionales y dinamizar los estudios. Aparecen dos modalidades: la movilidad como factor de cohesión de los mundos ibéricos y el análisis comparativo. Gracias a este último, en la revista se reconsideran las conquistas españolas de América, hasta el grado de presentarlas como la implementación de sistemas de dominio indirecto basados en la subsistencia de prácticas y grupos de poder prehispánicos hasta más allá del primer siglo del dominio hispano. ${ }^{70}$

La movilidad y la circulación de hombres, papeles y objetos entre los reinos y señoríos de esas monarquías también se ha revelado como importante propulsor de los estudios históricos. Las pautas de movilidad de la gente oriunda de Nueva España por los horizontes de la vasta Monarquía fueron estudiadas en un número de la revista en que se comparan los vínculos entre redes y grupos de manera simultánea en Hispanoamérica, España, los Países Bajos y el Extremo Oriente. ${ }^{71} \mathrm{O}$ bien se estudia la cuestión de la representación de cuerpos y de grupos ante la Corte del rey, llámense pueblos de indios u otras entidades como la Real Hacienda por medio de oficiales, agentes, procuradores y demás personeros. ${ }^{72}$

69 Remito a la sección monográfica del número 73 de Relaciones (1998), “La Monarquía española, grupos políticos locales ante la corte de Madrid”.

70 García Martínez, “Encomenderos españoles y British Residents” (240), 2011, pp. 1915-1978.

71 Rubial, "Religiosos viajeros” (243), 2012, pp. 813-848; Oropeza, “La esclavitud asiática” (241), 2011, pp. 5-57; Ruiz Ibáñez y Vallejo Cervantes, "Vivir 'sin dexar parte"” (243), 2012, pp. 1109-1170; Herrera Reviriego, "Flujos comerciales" (262), 2016, pp. 495-553.

72 Díaz Serrano, “La República de Tlaxcala” (243), 2012, pp. 1049-1107. 
En esta etapa destacan varias generaciones de investigadores: profesores insignes de El Colegio de México y de la Universidad Nacional, algunos de los cuales figuraban medio siglo antes como estudiantes aventajados. También están presentes investigadores en proceso de maduración. Proceden de casas de estudios como la Universidad Autónoma Metropolitana o el Instituto Nacional de Antropología e Historia. Con todo, en este pasado reciente son también numerosos los autores extranjeros, sobre todo docentes de universidades francesas, españolas y estadounidenses. Esto es reflejo del proceso de internacionalización de los estudios históricos antes mencionado.

\section{RECAPITULACIÓN}

De este recorrido por los artículos publicados en Historia Mexicana acerca de los siglos XVI y xvII se desprenden consideraciones que atañen al proceso de profesionalización de nuestra disciplina. Los artículos surgen de tres matrices o ámbitos: la interacción con el resto de las humanidades (1951-1970), la interlocución con las ciencias sociales con énfasis en la historia regional (1971-2001) y la formulación de nuevos lenguajes en un mundo globalizado (2002-2016).

Hay temas que reaparecen a todo lo largo. Su interés permanente vertebra de alguna manera la revista en lo tocante a los siglos aquí examinados: el ideario del padre Las Casas cuyo entorno es la controversia sobre la legitimidad del dominio del rey de España y los derechos de los indios como súbditos; los actores de la cristianización, en particular los frailes y las corrientes de pensamiento como el erasmismo y el milenarismo. Está el tema concerniente a las propias poblaciones autóctonas: demografía, pueblos, gobierno, religiosidad, historia, hispanización o aculturación, trabajo y fiscalidad. También sobresale la economía de los grupos no indios, sus actividades extractivas, tecnológicas, sus tendencias comerciales y la ruta transpacífica. 
Son las corporaciones o instituciones de índole eclesiástica otro de los temas recurrentes, con horizontes tan vastos como la historia social, pues encuentran ahí cabida cuestiones jurídicas, religiosas, científicas y educativas. Las regiones más estudiadas para los siglos aquí analizados son la Mixteca, Yucatán, Oaxaca central, Michoacán y Chiapas; enseguida vienen las aglomeraciones mineras del Septentrión de Nueva España: San Luis Potosí, Zacatecas, Durango. Por último, cabe destacar la relevancia, aunque no sean numerosos, de los trabajos que comparan o asocian la Nueva España con el Perú. Más allá, se advierten énfasis que vinculan a aquélla con Guatemala, las Filipinas y el Perú.

Como cabe esperar, por lo que hace a los siglos XVI y xvII, Historia Mexicana es una revista aún especializada en Nueva España. No hay trabajos que prescindan enteramente de ese reino a expensas de otra latitud, dominio o territorio. No obstante, a lo largo de sus 70 años, además de acoger autores procedentes de muy diversas latitudes, nuestra revista ha respondido con suma originalidad a las tendencias, métodos, debates e intereses más sobresalientes de la historiografía. En este sentido, su nombre no desdice de la proyección que hace de ella el importante referente internacional de todos conocido. 